\title{
Kawasaki Disease-Like Features in 10 Pediatric COVID-19 Cases: A Retrospective Study
}

Noor Ul Falah ${ }^{1}$, Shahnawaz Hashmi ${ }^{1}$, Zahoor Ahmed ${ }^{1}$, Ali Jaan ${ }^{1}$, Ali Akhtar ${ }^{2}$, Farhan Khalid ${ }^{1}$, Umar Farooque $^{3}$, Muhammad Tayyab Shera ${ }^{1}$, Sundas Ali ${ }^{4}$, Ayesha Javed ${ }^{1}$

1. Internal Medicine, Mayo Hospital, King Edward Medical University, Lahore, PAK 2. Internal Medicine, Army Medical College, National University of Medical Sciences, Rawalpindi, PAK 3. Neurology, Dow University of Health Sciences, Karachi, PAK 4. Internal Medicine, Holy Family Hospital, Rawalpindi Medical University, Rawalpindi, PAK

Corresponding author: Shahnawaz Hashmi, shahnawazhashmi76@gmail.com

\begin{abstract}
Children with coronavirus disease 2019 (COVID-19), which is caused by severe acute respiratory syndrome coronavirus type 2 (SARS-CoV-2), usually present with symptoms of mild upper respiratory tract infection without developing any significant complications. However, this observation has been rendered incautious by hundreds of clinical cases from around the world that have depicted a less benign multisystem inflammatory illness mimicking Kawasaki disease in COVID-positive pediatric patients. Our study aimed at retrospectively reviewing the different features of Kawasaki disease-like illness in children suffering from COVID-19, including the complications, laboratory investigations, treatment strategies used during their hospital stay, and outcomes. We searched the electronic database of the two pediatric units of Mayo Hospital, Lahore, Pakistan, for children who had been admitted to the ward between April 2020 and July 2020 and were diagnosed with COVID-19. A total of 10 such pediatric cases were found, whose clinical details were then reviewed and the obtained data were presented in the form of tables and percentages. The median age was between 4 months to 11 years (mean: 6 years). Of the 10 patients, 8 (80\%) were boys. Criteria for Kawasaki disease were met in all of them (100\%), with a complete presentation in five (50\%). Fever (100\%), conjunctival and oral cavity changes (90\%), and rash (80\%) were the most common features. Seven (70\%) patients required admission to a critical care unit, but no mortality occurred. This article can assist in understanding and dealing with Kawasaki disease-like manifestation of pediatric COVID-19 infection, especially in critical care settings, and its possible complications. It will help in a timely and appropriate decision-making regarding treatment and management of such cases.
\end{abstract}

Review began 10/07/2020 Review ended 10/14/2020 Published 10/19/2020

\section{○) Copyright 2020}

Falah et al. This is an open access article distributed under the terms of the Creative Commons Attribution License CC-BY 4.0., which permits unrestricted use, distribution, and reproduction in any medium, provided the original author and source are credited.
Categories: Internal Medicine, Pediatrics, Infectious Disease

Keywords: coronavirus disease, covid-19, children, sars-cov-2, coronavirus, respiratory tract infection, pediatric, kawasaki disease, inflammatory illness

\section{Introduction}

In 1967, Tomisaku Kawasaki described Kawasaki disease (KD) as a rare systemic vasculitis occurring in children $\geqslant 5$ years of age. Its etiology is still unknown, but an infectious trigger has been proposed. The diagnostic criteria for KD set by the American Heart Association include the following parameters: $\geqslant 5$ days of fever, oral mucosal changes, bilateral nonexudative conjunctivitis, exanthematous rash, desquamation of hands and feet, and cervical lymphadenopathy. Apart from hyperthermia, at least four of the five major clinical features are required for complete and less than four for an incomplete form of KD [1].

Since its outbreak in December 2019, the severe acute respiratory syndrome coronavirus type 2 (SARS-CoV2) infection has caused 30,624,590 confirmed cases and 953,903 confirmed deaths globally as of September 20, 2020 [2]. An epidemiological study from China showed that $90 \%$ of the 731 laboratory-confirmed COVID19 patients aged $<18$ years had asymptomatic, mild, or moderate infection, thus rendering it relatively benign than the adult infection [3]. Children with symptomatic COVID-19 may develop fever, fatigue, cough, sore throat, rhinorrhea, congestion, and shortness of breath, and the critical cases present with gastrointestinal distress, shock, and respiratory failure, deranged coagulation, and renal shutdown [4]. However, there has been an increase in the incidence of KD-like illness among COVID-positive children, either concomitant with or after the resolution of COVID-19 infection [5]. Some cases develop a shock-like systemic inflammatory illness resembling toxic shock syndrome, which is identified as a multisystem inflammatory syndrome, warranting their admission to the critical care unit [6].

\section{Materials And Methods}

We searched the electronic medical records (EMR) of the two pediatric units at Mayo Hospital, Lahore, Pakistan. Our search was limited to the pediatric COVID-19 patients admitted between April 2020 and July 2020. SARS-CoV-2 infection was confirmed on either a real-time reverse transcriptase polymerase chain reaction (RT-PCR) or a positive serology. A total of 10 such cases were found, whose clinical details were then thoroughly reviewed. Inclusion criteria involve the children aged 0-16 years diagnosed with COVID19. We studied the patients' demographics, comorbidities, signs, and symptoms of their COVID-19 infection, 


\section{Cureus}

including those of KD, complications, laboratory investigations, treatment strategies utilized in the hospital, and outcomes. One of the included cases had negative PCR and serology, but COVID-19 was diagnosed based on his symptoms, a history of COVID-19 contact, and CT results suggestive of SARS-CoV-2 pneumonia. Another PCR-negative case was suspected of having a past asymptomatic COVID-19 infection because of his locality and travel history of his father. The results were then presented as tables and percentages.

\section{Results}

A total of 10 pediatric cases with a median age of 4 months to 11 years (mean: 6 years) were included. Eight of them (80\%) were boys.

Criteria for KD were met in all of them (100\%), with a complete presentation in five (50\%) cases (Table 1 ). None reported any previous comorbidity. Polymerase chain reaction (PCR) test for SARS-CoV-2 was positive in seven (70\%) patients, whereas one patient (10\%) showed positive SARS-CoV-2 IgG serology. One case had negative PCR and serology, but COVID-19 was diagnosed on symptoms, history of COVID-19 contact, and lung CT, which revealed ground-glass opacities and consolidation in the right posterior basal area $(<10 \%$ of the lung parenchyma), suggestive of COVID-19 pneumonia. Another PCR-negative case was suspected of having a past asymptomatic COVID-19 infection because of father's locality and travel history.

\begin{tabular}{|c|c|c|c|c|c|c|c|c|c|c|}
\hline Characteristics & Patient 1 & Patient 2 & Patient 3 & Patient 4 & Patient 5 & Patient 6 & Patient 7 & Patient 8 & Patient 9 & $\begin{array}{l}\text { Patient } \\
10\end{array}$ \\
\hline Age and gender & $\begin{array}{l}\text { 5-year- } \\
\text { old boy }\end{array}$ & $\begin{array}{l}\text { 3-year- } \\
\text { old boy }\end{array}$ & $\begin{array}{l}\text { 10-year- } \\
\text { old boy }\end{array}$ & $\begin{array}{l}\text { 11-year- } \\
\text { old girl }\end{array}$ & $\begin{array}{l}\text { 6-month- } \\
\text { old girl }\end{array}$ & $\begin{array}{l}\text { 8-year- } \\
\text { old boy }\end{array}$ & $\begin{array}{l}\text { 4-month- } \\
\text { old boy }\end{array}$ & $\begin{array}{l}\text { 5-year- } \\
\text { old boy }\end{array}$ & $\begin{array}{l}\text { 11-year- } \\
\text { old boy }\end{array}$ & $\begin{array}{l}\text { 6-year- } \\
\text { old boy }\end{array}$ \\
\hline $\begin{array}{l}\text { Criteria met for } \\
\text { Kawasaki disease }\end{array}$ & Yes & Yes & Yes & Yes & Yes & Yes & Yes & Yes & Yes & Yes \\
\hline positive & Yes & No & Yes & Yes & Yes & Yes & Yes & Yes & No & Yes \\
\hline $\begin{array}{l}\text { SARS-CoV-2 serology } \\
\text { positive }\end{array}$ & Not done & Not done & Not done & Not done & Not done & Not done & Not done & Not done & positive & Not done \\
\hline Sick contacts & N/A & Yes & N/A & N/A & N/A & N/A & N/A & N/A & No & Yes \\
\hline Travel history & N/A & No & N/A & N/A & N/A & N/A & N/A & N/A & Yes & N/A \\
\hline Any comorbidity & No & No & No & No & No & No & No & No & No & No \\
\hline
\end{tabular}

TABLE 1: Patient characteristics

IgG, immunoglobulin G; N/A, not available

The symptoms of KD included fever $(\mathrm{n}=10)(100 \%)$, conjunctival and oral cavity changes (90\%), rash $(80 \%)$, extremity changes (50\%), and cervical lymphadenopathy (30\%) (Table 2). 


\section{Cureus}

\begin{tabular}{|c|c|c|c|c|c|c|c|c|c|c|}
\hline Kawasaki Feature & $\begin{array}{l}\text { Patient } \\
1\end{array}$ & $\begin{array}{l}\text { Patient } \\
2\end{array}$ & $\begin{array}{l}\text { Patient } \\
3\end{array}$ & $\begin{array}{l}\text { Patient } \\
4\end{array}$ & $\begin{array}{l}\text { Patient } \\
5\end{array}$ & $\begin{array}{l}\text { Patient } \\
6\end{array}$ & $\begin{array}{l}\text { Patient } \\
7\end{array}$ & $\begin{array}{l}\text { Patient } \\
8\end{array}$ & $\begin{array}{l}\text { Patient } \\
9\end{array}$ & $\begin{array}{l}\text { Patient } \\
10\end{array}$ \\
\hline $\begin{array}{l}\text { Complete presentation (fever }>4 \text { days and } \\
\geq 4 \text { principal criteria) }\end{array}$ & No & Yes & No & No & Yes & Yes & Yes & No & No & Yes \\
\hline Fever with duration & $\begin{array}{l}\text { Yes, } 8 \\
\text { days }\end{array}$ & $\begin{array}{l}\text { Yes, } 8 \\
\text { days }\end{array}$ & $\begin{array}{l}\text { Yes, } 7 \\
\text { days }\end{array}$ & $\begin{array}{l}\text { Yes, }>4 \\
\text { days }\end{array}$ & $\begin{array}{l}\text { Yes, }>4 \\
\text { days }\end{array}$ & $\begin{array}{l}\text { Yes, }>4 \\
\text { days }\end{array}$ & $\begin{array}{l}\text { Yes, }>4 \\
\text { days }\end{array}$ & $\begin{array}{l}\text { Yes, }>4 \\
\text { days }\end{array}$ & $\begin{array}{l}\text { Yes, }>4 \\
\text { days }\end{array}$ & $\begin{array}{l}\text { Yes, }>7 \\
\text { days }\end{array}$ \\
\hline Rash & No & Yes & Yes & Yes & Yes & Yes & Yes & No & Yes & Yes \\
\hline Conjunctival injection & Yes & Yes & Yes & No & Yes & Yes & Yes & Yes & Yes & Yes \\
\hline Lips and oral cavity changes & Yes & Yes & Yes & Yes & Yes & Yes & Yes & No & Yes & Yes \\
\hline Cervical Lymphadenopathy & Yes & Yes & No & No & No & No & Yes & No & No & No \\
\hline Extremity changes (swelling, erythema) & No & No & No & Yes & Yes & Yes & No & Yes & No & Yes \\
\hline
\end{tabular}

TABLE 2: Features of Kawasaki disease

Among other symptoms, gastrointestinal problems were prevalent (70\%) followed by neurological (30\%) and respiratory dysfunctions (20\%). Among the gastrointestinal symptoms, diarrhea and abdominal pain were most common $(n=5)(50 \%)$. Three (30\%) cases developed perineal, periungal, or facial desquamation (Table 3). 


\section{Cureus}

\begin{tabular}{|c|c|c|c|c|c|c|c|c|c|c|}
\hline Symptoms and Signs & $\begin{array}{l}\text { Patient } \\
1\end{array}$ & $\begin{array}{l}\text { Patient } \\
2\end{array}$ & $\begin{array}{l}\text { Patient } \\
3\end{array}$ & $\begin{array}{l}\text { Patient } \\
4\end{array}$ & $\begin{array}{l}\text { Patient } \\
5\end{array}$ & $\begin{array}{l}\text { Patient } \\
6\end{array}$ & $\begin{array}{l}\text { Patient } \\
7\end{array}$ & $\begin{array}{l}\text { Patient } \\
8\end{array}$ & $\begin{array}{l}\text { Patient } \\
9\end{array}$ & $\begin{array}{l}\text { Patient } \\
10\end{array}$ \\
\hline Sore throat & No & No & No & Yes & No & Yes & No & No & No & Yes \\
\hline Respiratory symptoms & No & No & Yes & No & No & Yes & No & No & No & No \\
\hline Cough & - & - & Yes & - & - & Yes & - & - & - & - \\
\hline Dyspnea & - & - & Yes & - & - & Yes & - & - & - & - \\
\hline Stuffy nose/sneezing/rhinorrhea & - & - & - & - & - & - & - & Yes & Yes & Yes \\
\hline GI symptoms & Yes & No & Yes & Yes & Yes & No & No & Yes & Yes & Yes \\
\hline Abdominal pain & Yes & - & No & Yes & No & - & - & Yes & Yes & Yes \\
\hline Diarrhea & Yes & - & Yes & No & No & - & - & No & No & Yes \\
\hline Vomiting & No & - & No & No & No & - & - & No & No & No \\
\hline Decreased appetite & Yes & - & No & Yes & Yes & - & - & No & No & No \\
\hline Dysuria & Yes & No & No & No & No & No & No & Yes & No & No \\
\hline Neurological symptoms & No & No & No & No & Yes & No & Yes & No & - & - \\
\hline Irritability & - & - & - & - & Yes & No & Yes & No & - & - \\
\hline Anosmia & - & - & - & - & No & No & No & No & - & - \\
\hline Headache & - & - & - & - & No & No & No & No & - & - \\
\hline Confusion & - & - & - & -- & No & No & No & No & - & - \\
\hline Meningeal signs & - & - & - & - & No & No & No & Yes & - & - \\
\hline Drowsiness & - & - & - & - & No & No & No & Yes & - & - \\
\hline $\begin{array}{l}\text { Perineal/periungal/facial } \\
\text { desquamation }\end{array}$ & No & Yes & No & No & No & Yes & No & No & Yes & No \\
\hline
\end{tabular}

\section{TABLE 3: Symptoms and signs}

Gl, gastrointestinal

Complications included admission to a critical care unit (70\%), hypotension/shock (60\%), inotropic support (40\%), pericarditis/pericardial effusion (40\%), myocarditis (30\%), coronary artery dilation (20\%), acute kidney injury (10\%), paralytic ileus (10\%), and coronary aneurysm in none (Table 4$)$. None of them required artificial ventilation, and no mortality was reported in any case except for one whose outcome data was not available. 


\begin{tabular}{|c|c|c|c|c|c|c|c|c|c|c|}
\hline Complication & $\begin{array}{l}\text { Patient } \\
1\end{array}$ & $\begin{array}{l}\text { Patient } \\
2\end{array}$ & $\begin{array}{l}\text { Patient } \\
3\end{array}$ & $\begin{array}{l}\text { Patient } \\
4\end{array}$ & $\begin{array}{l}\text { Patient } \\
5\end{array}$ & $\begin{array}{l}\text { Patient } \\
6\end{array}$ & $\begin{array}{l}\text { Patient } \\
7\end{array}$ & $\begin{array}{l}\text { Patient } \\
8\end{array}$ & Patient9 & $\begin{array}{l}\text { Patient } \\
10\end{array}$ \\
\hline $\begin{array}{l}\text { Admission to the critical care } \\
\text { unit }\end{array}$ & Yes & No & Yes & Yes & No & Yes & No & Yes & Yes & Yes \\
\hline Artificial ventilation & No & No & No & No & No & No & No & No & No & No \\
\hline Hypotension/shock & Yes & No & Yes & Yes & No & Yes & No & Yes & Yes & No \\
\hline Inotropic support & No & No & Yes & Yes & No & No & No & Yes & Yes & No \\
\hline Acute kidney injury & No & No & No & Yes & No & No & No & No & No & No \\
\hline Coronary artery dilatation & No & No & No & No & No & No & Yes & No & Yes & No \\
\hline Coronary artery aneurysm & No & No & No & No & No & No & No & No & No & No \\
\hline Myocarditis & No & No & Yes & Yes & No & No & No & Yes & No & No \\
\hline Pericardial effusion/pericarditis & Yes & No & Yes & No & No & No & No & No & Yes & No \\
\hline Paralytic ileus & No & No & No & No & No & No & No & No & No & Yes \\
\hline Mortality & No & No & $\mathrm{N} / \mathrm{a}$ & No & No & No & No & No & No & No \\
\hline
\end{tabular}

\section{TABLE 4: Complications}

N/A, not available

All the cases reported elevated C-reactive protein levels, whereas raised levels of ferritin (60\%), erythrocyte sedimentation rate (ESR) (50\%), procalcitonin (50\%), and interleukin-6 (10\%) were also noted (Table 5). Other significant findings included leukocytosis (90\%) and hypoalbuminemia (70\%). Raised levels of fibrinogen (40\%), D-dimers (30\%), lactate dehydrogenase (LDH) (20\%), troponin (40\%), and N-terminal proB-type natriuretic peptide (NT-proBNP) (40\%), as well as anemia (50\%), thrombocytopenia (30\%), and lymphopenia (30\%) were seen. Moreover, six (60\%) cases had abnormal chest X-ray findings.

\begin{tabular}{|c|c|c|c|c|c|c|c|c|c|c|}
\hline $\begin{array}{l}\text { Inflammatory } \\
\text { Markers }\end{array}$ & Patient 1 & $\begin{array}{l}\text { Patient } \\
2\end{array}$ & $\begin{array}{l}\text { Patient } \\
3\end{array}$ & $\begin{array}{l}\text { Patient } \\
4\end{array}$ & Patient 5 & Patient 6 & Patient 7 & Patient 8 & Patient 9 & Patient 10 \\
\hline $\begin{array}{l}\text { CRP (0-5 } \\
\mathrm{mg} / \mathrm{L})\end{array}$ & $\begin{array}{l}\text { Raised (250 } \\
\mathrm{mg} / \mathrm{L})\end{array}$ & $\begin{array}{l}\text { raised } \\
(195 \\
\mathrm{mg} / \mathrm{L})\end{array}$ & $\begin{array}{l}\text { Raised } \\
(280 \\
\mathrm{mg} / \mathrm{L})\end{array}$ & $\begin{array}{l}\text { Raised } \\
\text { (>300 } \\
\mathrm{mg} / \mathrm{L})\end{array}$ & $\begin{array}{l}\text { Raised } \\
\text { (133 mg/L) }\end{array}$ & $\begin{array}{l}\text { Raised } \\
\text { (317 mg/L) }\end{array}$ & $\begin{array}{l}\text { Raised } \\
\text { (115.6 } \\
\text { mg/L) }\end{array}$ & $\begin{array}{l}\text { Raised (120 } \\
\mathrm{mg} / \mathrm{L})\end{array}$ & $\begin{array}{l}\text { Raised (189.5 } \\
\mathrm{mg} / \mathrm{L})\end{array}$ & $\begin{array}{l}\text { Raised (130 } \\
\mathrm{mg} / \mathrm{L})\end{array}$ \\
\hline $\begin{array}{l}\text { Ferritin (7- } \\
140 \mu \mathrm{g} / \mathrm{L})\end{array}$ & $\begin{array}{l}\text { Raised (1030 } \\
\mu \mathrm{g} / \mathrm{L})\end{array}$ & N/A & $\begin{array}{l}\text { Raised } \\
(1089 \\
\mu g / L)\end{array}$ & $\begin{array}{l}\text { Raised } \\
\text { (1789 } \\
\mu \mathrm{g} / \mathrm{L})\end{array}$ & N/A & $\begin{array}{l}\text { Raised } \\
(1496 \\
\mu \mathrm{g} / \mathrm{L})\end{array}$ & $\mathrm{N} / \mathrm{A}$ & $\begin{array}{l}\text { Raised (600 } \\
\mu \mathrm{g} / \mathrm{L} \text { ) }\end{array}$ & N/A & $\begin{array}{l}\text { Raised (612 } \\
\mu \mathrm{g} / \mathrm{L})\end{array}$ \\
\hline $\begin{array}{l}\text { ESR (0-22 } \\
\mathrm{mm} / \mathrm{hr})\end{array}$ & $\begin{array}{l}\text { Raised (72 } \\
\mathrm{mm} / \mathrm{hr})\end{array}$ & N/A & $\begin{array}{l}\text { Raised } \\
\text { (57) }\end{array}$ & N/A & $\begin{array}{l}\text { Raised } \\
\text { (118) }\end{array}$ & $\begin{array}{l}\text { Raised } \\
\text { (115) }\end{array}$ & $\mathrm{N} / \mathrm{A}$ & Raised (70) & N/A & N/A \\
\hline $\begin{array}{l}\text { Procalcıtonın } \\
\text { (<2 } \mu \mathrm{g} / \mathrm{L})\end{array}$ & $\begin{array}{l}\text { Ralsed (27 } \\
\mu \mathrm{g} / \mathrm{L})\end{array}$ & $\mathrm{N} / \mathrm{A}$ & $\begin{array}{l}\text { Raised } \\
(28 \\
\mu g / L)\end{array}$ & $\begin{array}{l}\text { Raised } \\
\text { (16.28 } \\
\mu \mathrm{g} / \mathrm{L})\end{array}$ & N/A & N/A & N/A & N/A & $\begin{array}{l}\text { Ralsed (14.55 } \\
\mu \mathrm{g} / \mathrm{L})\end{array}$ & $\begin{array}{l}\text { Raised (5.05 } \\
\mu \mathrm{g} / \mathrm{L})\end{array}$ \\
\hline $\begin{array}{l}\text { IL-6 (<8.5 } \\
\mathrm{pg} / \mathrm{mL})\end{array}$ & N/A & N/A & N/A & $\begin{array}{l}\text { Raised } \\
\text { (1449 } \\
\text { pg/mL) }\end{array}$ & N/A & N/A & $\mathrm{N} / \mathrm{A}$ & N/A & N/A & N/A \\
\hline \multicolumn{11}{|c|}{ Coagulation profile } \\
\hline $\begin{array}{l}\text { D-dimers } \\
(100-560 \\
\mathrm{ng} / \mathrm{mL})\end{array}$ & N/A & N/A & $\begin{array}{l}\text { Raised } \\
\text { (2727 } \\
\mathrm{ng} / \mathrm{mL})\end{array}$ & $\begin{array}{l}\text { Raised } \\
\text { (1207 } \\
\text { ng/mL) }\end{array}$ & N/A & N/A & $\mathrm{N} / \mathrm{A}$ & N/A & $\begin{array}{l}\text { raised (894 } \\
\mathrm{ng} / \mathrm{mL})\end{array}$ & N/A \\
\hline $\begin{array}{l}\text { Fibrinogen } \\
(1.99-4.09 \\
\text { g/L) }\end{array}$ & N/A & N/A & $\begin{array}{l}\text { Raised } \\
(7.48 \\
\text { g/L) }\end{array}$ & $\begin{array}{l}\text { Raised } \\
(597 \\
\text { g/L) }\end{array}$ & N/A & N/A & $\mathrm{N} / \mathrm{A}$ & N/A & $\begin{array}{l}\text { Ralsed (18.61 } \\
\mathrm{g} / \mathrm{L})\end{array}$ & Raised (5.2 \\
\hline
\end{tabular}




\section{Cureus}

\begin{tabular}{|c|c|c|c|c|c|c|c|c|c|c|}
\hline \multicolumn{11}{|l|}{ Biochemistry } \\
\hline $\begin{array}{l}\text { LDH (125-243 } \\
\text { U/L) }\end{array}$ & N/A & N/A & $\begin{array}{l}\text { Raised } \\
(360 \\
\text { U/L) }\end{array}$ & $\begin{array}{l}\text { Raised } \\
\text { (301 } \\
\text { U/L) }\end{array}$ & N/A & N/A & N/A & N/A & N/A & N/A \\
\hline $\begin{array}{l}\text { Albumin (35- } \\
54 \mathrm{~g} / \mathrm{L})\end{array}$ & $\begin{array}{l}\text { Decreased } \\
(20 \mathrm{~g} / \mathrm{L})\end{array}$ & N/A & N/A & N/A & $\begin{array}{l}\text { Decreased } \\
(28 \mathrm{~g} / \mathrm{L})\end{array}$ & $\begin{array}{l}\text { Decreased } \\
(26 \mathrm{~g} / \mathrm{L})\end{array}$ & $\begin{array}{l}\text { Decreased } \\
(30 \mathrm{~g} / \mathrm{L})\end{array}$ & $\begin{array}{l}\text { Decreased } \\
(21 \mathrm{~g} / \mathrm{L})\end{array}$ & $\begin{array}{l}\text { Decreased } \\
(22 \mathrm{~g} / \mathrm{L})\end{array}$ & $\begin{array}{l}\text { Decreased (27 } \\
\text { g/L) }\end{array}$ \\
\hline \multicolumn{11}{|c|}{ Cardiac markers } \\
\hline $\begin{array}{l}\text { Troponin (0- } \\
15 \text { ng/L) }\end{array}$ & $\begin{array}{l}\text { Raised (60 } \\
\text { ng/L) }\end{array}$ & N/A & $\begin{array}{l}\text { Raised } \\
(84 \\
\mathrm{ng} / \mathrm{L})\end{array}$ & $\begin{array}{l}\text { Raised } \\
112 \\
\mathrm{ng} / \mathrm{L})\end{array}$ & N/A & $\mathrm{N} / \mathrm{A}$ & N/A & $\begin{array}{l}\text { Raised (29 } \\
\text { ng/L) }\end{array}$ & normal & N/A \\
\hline $\begin{array}{l}\text { NT-proBNP } \\
(<300 \text { pg/mL) }\end{array}$ & N/A & $\mathrm{N} / \mathrm{A}$ & $\begin{array}{l}\text { Raised } \\
\text { (9477 } \\
\mathrm{pg} / \mathrm{mL})\end{array}$ & $\begin{array}{l}\text { Raised } \\
\text { (8718 } \\
\mathrm{pg} / \mathrm{mL})\end{array}$ & N/A & N/A & N/A & $\begin{array}{l}\text { Raised (8000 } \\
\mathrm{pg} / \mathrm{mL})\end{array}$ & $\begin{array}{l}\text { Raised (3131 } \\
\mathrm{pg} / \mathrm{mL})\end{array}$ & $\mathrm{N} / \mathrm{A}$ \\
\hline \multicolumn{11}{|l|}{ Blood profile } \\
\hline $\begin{array}{l}\text { White cell } \\
\text { count (4-15.5 } \\
\left.\times 10^{9}\right)\end{array}$ & Raised & Raised & Raised & Raised & Raised & Raised & Raised & Raised & normal & Slightly raised \\
\hline Lymphopenia & N/A & N/A & yes & yes & N/A & N/A & N/A & yes & N/A & N/A \\
\hline $\begin{array}{l}\text { Hemoglobin } \\
\text { (111-147 g/L) }\end{array}$ & Decreased & N/A & Normal & N/A & Decreased & Decreased & Decreased & N/A & N/A & $\begin{array}{l}\text { Slightly } \\
\text { decreased }\end{array}$ \\
\hline $\begin{array}{l}\text { Platelets } \\
(200-450 x \\
\left.10^{9}\right)\end{array}$ & Decreased & N/A & Normal & Normal & Normal & Normal & Normal & Normal & Decreased & Decreased \\
\hline Chest X-ray & Cardiomegaly & N/A & N/A & N/A & $\begin{array}{l}\text { Faint } \\
\text { opacity in } \\
\text { the left } \\
\text { mid-lung } \\
\text { zone }\end{array}$ & $\begin{array}{l}\text { Right } \\
\text { upper and } \\
\text { middle } \\
\text { lobe } \\
\text { infiltrates }\end{array}$ & Normal & Cardiomegaly & Cardiomegaly & $\begin{array}{l}\text { Pulmonary } \\
\text { infiltrates at the } \\
\text { right base with } \\
\text { accentuated } \\
\text { bronchovascular } \\
\text { markings in } \\
\text { bilateral perihilar } \\
\text { and para- } \\
\text { cardiac regions }\end{array}$ \\
\hline
\end{tabular}

\section{TABLE 5: Hospital Investigations}

CRP, C-reactive protein; ESR, erythrocyte sedimentation rate; IL-6, interleukin-6; LDH, lactate dehydrogenase; N/A, not available; NT-proBNP, Nterminal pro-B-type natriuretic peptide

Treatment strategies focused on the management of KD-like features as well as the symptomatic treatment of COVID-19 (Table 6). For KD-like illness, 9 (90\%) out of 10 cases received intravenous immunoglobulin (IVIG) therapy, whereas 7 (70\%) of them received acetylsalicylic acid as well. The symptomatic treatment for COVID-19 included antibiotics (60\%), oxygen (40\%), steroids (30\%), tocilizumab (20\%), anticoagulation, plasma therapy, and antivirals (10\%). 


\begin{tabular}{|c|c|c|c|c|c|c|c|c|c|c|}
\hline Treatment & Patient 1 & Patient 2 & Patient 3 & Patient 4 & Patient 5 & Patient 6 & Patient 7 & Patient 8 & Patient 9 & Patient 10 \\
\hline IVIG therapy & Yes & Yes & $\mathrm{N} / \mathrm{a}$ & Yes & Yes & Yes & Yes & Yes & Yes & Yes \\
\hline Acetylsalicylic acid & Yes & No & $\mathrm{N} / \mathrm{a}$ & No & Yes & Yes & Yes & Yes & Yes & Yes \\
\hline Oxygen & Yes & No & $\mathrm{N} / \mathrm{a}$ & No & No & Yes & No & Yes & No & Yes \\
\hline Steroids & Yes & No & $\mathrm{N} / \mathrm{a}$ & Yes & No & No & No & Yes & No & No \\
\hline Antibiotics & No & No & $\mathrm{N} / \mathrm{a}$ & Yes & No & Yes & Yes & Yes & Yes & Yes \\
\hline Tocilizumab & No & No & $\mathrm{N} / \mathrm{a}$ & Yes & No & Yes & No & No & No & No \\
\hline Anticoagulant & No & No & $\mathrm{N} / \mathrm{a}$ & Yes & No & No & No & No & No & No \\
\hline Antiviral therapy & No & No & $\mathrm{N} / \mathrm{a}$ & Yes & No & No & No & No & No & No \\
\hline Plasma therapy & No & No & $\mathrm{N} / \mathrm{a}$ & Yes & No & No & No & No & No & No \\
\hline
\end{tabular}

\section{TABLE 6: Treatment strategies}

IVIG, intravenous immunoglobulin

\section{Discussion}

$\mathrm{KD}$ is an acute and self-limiting vasculitis that is characterized by the inflammation of the medium caliber vessels. It almost exclusively affects children [7]. In the acute phase, patients with KD might develop hemodynamic instability, a condition known as KD shock syndrome (KDSS) [8]. KD is the chief cause of acquired heart disease among children in developed countries resulting in detrimental complications such as coronary artery ectasias, cardiac inflammation, and aneurysms in 15-25\% of patients if left untreated. KD may also cause myocarditis, pericarditis, and abnormal heart rhythms [1].

There are published studies regarding the presence of KD-like features in pediatric COVID-19 infection. The pathophysiology of KD and COVID-19 is similar, i.e., exaggerated inflammatory response (cytokine storm) leading to vascular endothelial damage and immune-mediated tissue injury. This may account for the overlapping of their symptoms [9]. COVID-19 patients also demonstrate KD-like coronary changes, as seen in two (20\%) of the cases included in our study who developed coronary artery dilation. None of the patients developed a coronary aneurysm. Pericardial effusion was seen in four (40\%) patients and myocarditis was seen in three (30\%) patients. In another study, 19 (90\%) out of 21 children and adolescents (median age: 7.9 years) who were admitted with features of KD over 15 days showed evidence of recent SARS-CoV-2 infection. Also, 12 (57\%) presented with KD shock syndrome and 16 (76\%) with myocarditis, with 17 (81\%) requiring intensive care support. All of them had significant gastrointestinal symptoms during the early stage of illness and increased inflammatory markers. Each one of them had a favorable clinical outcome. Moderate coronary artery dilations were detected in five (24\%) of the patients [10].

Typical KD has certain differences from the pattern seen in COVID-19 patients. Although KD can affect the pediatric population of any race, those with Asian origin are more prone to it [1]. This may suggest that in COVID-19 infection, KD-like symptoms can occur irrespective of the racial predisposition. Moreover, the cases included in our study were reported between April and July. This pattern is also different from the usual winter predisposition of KD, as indicated by the three KD epidemics recorded in Japan in 1979, 1982, and 1986, with the highest incidence seen in January [11]. In a study, the Kawasaki-COVID-19 cohort differed from a comparator group of 'classical' KD by older age at onset (10 vs 2 years; $\mathrm{p}<0.0001$ ), lower platelet count ( 188 vs. $383 \mathrm{G} / \mathrm{L} ; \mathrm{p}<0.0001)$, a higher rate of myocarditis (7/16 vs. 3/220; p=0.0001), and resistance to first IVIG treatment (10/16 vs. 45/220; $\mathrm{p}=0.004)$ [12].

The cause of KD remains unknown, although half a century has passed since KD's first case was reported [7]. The most accepted hypothesis supports an aberrant response of the immune system to one or more unidentified pathogens in genetically predisposed patients [13]. It is worth mentioning here that KD lacks a definitive association with any single etiological agent, and multiple infectious triggers such as rhinovirus, parainfluenza virus, respiratory syncytial virus, adenovirus [14], human coronavirus (HCoV-229E) [15], and new haven coronavirus (HCoV-NH) [16] have been linked to it. However, some studies have disproved the connection of human coronavirus with $\mathrm{KD}$ [17]. Hence, it is yet to be ascertained whether the detection of SARS-CoV-2 in children with associated KD symptoms should be treated under a diagnosis of KD, or a new separate diagnosis of COVID-19 infection is required. There is a need to differentiate an incidental SARS$\mathrm{CoV}-2$ infection with concomitant KD from KD triggered by SARS-CoV-2. 
Our study included only 10 cases, which limits its reliability due to the small sample size. However, KD criteria were met in all of them (100\%), with a complete presentation in 5 (50\%). Fever was present in all patients. The conjunctival and oral cavity changes, including rash, were the most common features. Seven (70\%) children required admission to a critical care unit, but no mortality occurred. This article helps understand and deal with KD-like manifestation of pediatric COVID-19 infection, especially in critical care settings, and its possible complications. It will help in timely and appropriate decision-making regarding treatment and management of such cases. More studies on a large scale are required to prove any association between pediatric COVID-19 infection and KD. Yet, to be on the safe side, it is advised that care should be taken when reintegrating these pediatric patients in the society.

\section{Conclusions}

The clinical spectrum of COVID-19 symptoms in children is yet to be defined, and no guidelines have been established regarding screening of COVID-positive pediatric patients for KD. Lack of timely diagnosis and management of concurrent KD can prove detrimental. Hence, the children diagnosed with KD should be tested for SARS-CoV-2, the pathogen for COVID-19, to differentiate the etiology from other infectious triggering agents and avoid missing underlying COVID-19.

\section{Additional Information \\ Disclosures}

Human subjects: Consent was obtained by all participants in this study. NA issued approval NA. NA. Animal subjects: All authors have confirmed that this study did not involve animal subjects or tissue. Conflicts of interest: In compliance with the ICMJE uniform disclosure form, all authors declare the following: Payment/services info: All authors have declared that no financial support was received from any organization for the submitted work. Financial relationships: All authors have declared that they have no financial relationships at present or within the previous three years with any organizations that might have an interest in the submitted work. Other relationships: All authors have declared that there are no other relationships or activities that could appear to have influenced the submitted work.

\section{References}

1. Freeman AF, Shulman ST: Kawasaki disease: summary of the American Heart Association guidelines. Am Fam Physician. 2006, 74:1141-1148.

2. WHO Coronavirus Disease (COVID-19) Dashboard. (2020). Accessed: September 10, 2020: https://covid19.who.int/.

3. Dong Y, Mo X, Hu Y, Qi X, Jiang F, Jiang Z, Tong S: Epidemiological characteristics of 2143 pediatric patients with 2019 coronavirus disease in China. Pediatrics. 2020, 1542:20200702. 10.1542/peds.2020-0702

4. Jones VG, Mills M, Suarez D, et al.: COVID-19 and Kawasaki disease: novel virus and novel case . Hosp Pediatr. 2020, 10:537-540. 10.1542/hpeds.2020-0123.

5. Sandhaus H, Crosby D, Sharma A, Gregory SR: Association between COVID-19 and Kawasaki disease: vigilance required from otolaryngologists. Otolaryngol Head Neck Surg. 2020, 163:316-317. 10.1177/0194599820930238

6. Feldstein LR, Rose EB, Horwitz SM, et al.: Multisystem inflammatory syndrome in US children and adolescents. N Engl J Med. 2020, 383:334-346. 10.1056/NEJMoa2021680

7. Kawasaki T, Kosaki F, Okawa S, Shigematsu I, Yanagawa H: A new infantile acute febrile mucocutaneous lymph node syndrome (MLNS) prevailing in Japan. Pediatrics. 1974, 54:271-276.

8. Kanegaye JT, Wilder MS, Molkara D, et al.: Recognition of a Kawasaki disease shock syndrome Pediatrics. 2009, 123:783-789. 10.1542/peds.2008-1871

9. Rauf A, Vijayan A, John ST, Krishnan R, Latheef A: Multisystem inflammatory syndrome with features of atypical Kawasaki disease during COVID-19 pandemic. Indian J Pediatr. 2020, 87:745-747. 10.1007/s12098020-03357-1

10. Toubiana J, Poirault C, Corsia A, et al.: Kawasaki-like multisystem inflammatory syndrome in children during the Covid-19 pandemic in Paris, France: prospective observational study. BMJ. 2020, 369:2094. 10.1136/bmj.m2094

11. Makino N, Nakamura Y, Yashiro M, et al.: Descriptive epidemiology of Kawasaki disease in Japan, 20112012: from the results of the 22nd nationwide survey. J Epidemiol. 2015, 25:239-245. 10.2188/jea.JE20140089

12. Pouletty M, Borocco C, Ouldali N, et al.: Paediatric multisystem inflammatory syndrome temporally associated with SARS-CoV-2 mimicking Kawasaki disease (Kawa-COVID-19): a multicentre cohort. Ann Rheum Dis. 2020, 79:999-1006. 10.1136/annrheumdis-2020-217960

13. Shulman ST, Rowley AH: Kawasaki disease: insights into pathogenesis and approaches to treatment . Nat Rev Rheumatol. 2015, 11:475-482. 10.1038/nrrheum.2015.54

14. Turnier JL, Anderson MS, Heizer HR, Jone PN, Glodé MP, Dominguez SR: Concurrent respiratory viruses and Kawasaki disease. Pediatrics. 2015, 136:609-614. 10.1542/peds.2015-0950

15. Shirato K, Imada Y, Kawase M, Nakagaki K, Matsuyama S, Taguchi F: Possible involvement of infection with human coronavirus 229E, but not NL63, in Kawasaki disease. J Med Virol. 2014, 86:2146-2153. 10.1002/jmv.23950

16. Esper F, Shapiro ED, Weibel C, Ferguson D, Landry ML, Kahn JS: Association between a novel human coronavirus and Kawasaki disease. J Infect Dis. 2005, 191:499-502. 10.1086/428291

17. Belay ED, Erdman DD, Anderson LJ, et al.: Kawasaki disease and human coronavirus. J Infect Dis. 2005, 192:352-353. 10.1086/431609 